\title{
On Fields with Finite Information Density
}

\author{
Achim Kempf \\ Departments of Applied Mathematics and Physics, University of Waterloo \\ Perimeter Institute for Theoretical Physics \\ Waterloo, Ontario, Canada \\ Email: akempf@uwaterloo.ca
}

\begin{abstract}
The existence of a natural ultraviolet cutoff at the Planck scale is widely expected. In a previous Letter, it has been proposed to model this cutoff as an information density bound by utilizing suitably generalized methods from the mathematical theory of communication. Here, we prove the mathematical conjectures that were made in this Letter.
\end{abstract}

\section{Introduction}

Gedanken experiments indicate that the basic spatial notions of length, area and volume lose operational meaning at the Planck scale of $10^{-35} \mathrm{~m}$ (assuming $3+1$ dimensions). The argument is that any attempt at resolving smaller scales would imply a momentum uncertainty large enough to cause a curvature uncertainty which would spoil the attempted spatial resolution. It has been widely suggested, therefore, that the uncertainty relations should contain corrections that imply a finite lower bound to the achievable uncertainty in localizing the position or time of events, see [1. If there exists such a smallest length, area or volume in nature this should imply some form of an upper bound to the information density that can be imprinted on physical fields. This is indicated as well by studies of holography, see e.g. [2].

The question arises, however, in which way such an upper bound to information density could be modelled mathematically. In [3], it was proposed to model the information density bound in essentially the same way that has been successful in standard information theory and its applications to communication engineering.

In the early days of communication engineering, the problem arose how to quantify the amount of information contained in continuous signals of music, speech or images. Claude Shannon solved the problem in two steps in his seminal work [6] which started modern information theory. First, he noticed that bandlimited continuous signals such 
as music are fully captured by discrete samples, i.e. that they are fully reconstructible from those samples, if the spacing of those samples does not exceed $1 / 2 \omega_{\max }$, where $\omega_{\max }$ is the bandwidth of the signal, i.e., the largest frequency that occurs in the signal. The mathematical discipline which studies the reconstruction of functions from samples is now known as Shannon sampling theory and this is what we here attempt to generalize for applications to Planck scale physics. The proposal is that physical fields could be captured everywhere if sampled only at discrete points in space, as long as the spacing of those sampling points is sufficiently small, say of the order of the Planck distance. As the second step, Shannon considered the information density limiting effect of noise in the signal. While important, we will here not consider this second issue, other than mentioning that the role of noise could be played by quantum fluctuations.

In order to generalize Shannon sampling theory for use in the physics of general curved spacetimes, it is important to develop a sampling theory that allows both the bandwidth and the density of samples to vary continuously: the original Shannon sampling theorem requires equidistantly-spaced samples but on generic curved spaces there are no equidistantly spaced lattices.

In [3], as a first step, a generalization of Shannon sampling theory for the case of one dimension was outlined and its main features were conjectured. On the basis of those conjectures, a new method of data compression that utilizes variable sampling rates (adapted to the signal) was developed and a patent has been granted, 4. Here, we will explicitly carry out the program which was outlined in [3], thereby proving the conjectures that were made in [3. In addition, we show that we are dealing with a reproducing kernel Hilbert space and we explicitly calculate its reproducing kernel.

Recently, in [5], this framework has been generalized to describe a covariant information-theoretic cutoff in arbitrary-dimensional curved spacetime. The results and techniques developed in the present paper should be very useful for explicitly carrying out the much more general program proposed in [5].

\section{Terminology and notation}

We are concerned with the theory of continuous functions that can be reconstructed from any discrete set of samples that is sufficiently tightly spaced. This field of research, sampling theory, is well-established both in mathematics and in communication engineering. While we are here targeting applications to Planck scale physics we will use the established terminology. The translation of terms is obvious: signals mean real scalar fields, bandwidth means ultraviolet cutoff, the time coordinate for signals could be both a time coordinate or a space coordinate for fields, etc. Note that in the case of fields, unlike in the case of signals, samples of field amplitudes are generally not directly measurable. Both in quantum mechanics and in quantum field theory the measurement problem is deep and unsolved. If discrete samples suffice to capture a 
field everywhere this may have profound implications on the measurement problem. While very interesting, we will here not further pursue this question. Also, we will be concerned with the sampling theory of scalar fields only. The generalization to vector, spinor and operator-valued fields will be considered elsewhere.

Let us begin by noting that the mathematical language of the generalized sampling theory that we are concerned with is also the mathematical language of quantum mechanics, namely functional analysis. While this suggests the use Dirac's bra-ket notation, there are good reasons for using the proper mathematical notation instead. For example, instead of Dirac's $\langle\phi|A| \psi\rangle$ we will write $(\phi, A \psi)$. The reason is that we will have to be precise about the domains and adjoints of operators. While an expression such as $(A \phi, \psi)$ is unambiguous, the same expression using Dirac's notation would require bracketing which becomes exceedingly cumbersome in more complicated situations.

\section{Shannon sampling theory}

We begin by recalling elements of Shannon sampling theory. The basic Shannon sampling theorem states that in order to capture a signal $\phi(t)$ with bandwidth $\omega_{\max }$ for all times $t$, it is sufficient to record only the signals' values at the discrete points in time $\left\{t_{n}\right\}$ with spacing $s=t_{n+1}-t_{n}$, where:

$$
s=\frac{1}{2 \omega_{\max }}
$$

If the samples are taken at this rate, which is the so-called Nyquist rate, then the Shannon sampling formula allows the reconstruction of the continuous signal $\phi(t)$ at all times $t$ from its values $\phi\left(t_{n}\right)$ at the discrete times $\left\{t_{n}\right\}$ :

$$
\phi(t)=\sum_{n=-\infty}^{+\infty} \frac{\sin \left(2 \pi\left(t-t_{n}\right) \omega_{\max }\right)}{2 \pi\left(t-t_{n}\right) \omega_{\max }} \phi\left(t_{n}\right)
$$

There is a 1-parameter family of "sampling time lattices" $\left\{t_{n}(\alpha)\right\}$ which all have the required spacing $s$, namely

$$
t_{n}(\alpha)=\frac{n}{2 \omega_{\max }}+\alpha
$$

where $\alpha \in\left[0,1 / 2 \omega_{\max }\right]$. The sampling formula Eq,2 applies equally to all of these sampling lattices.

The sampling theorem has a simple proof which combines discrete and continuous Fourier transforms. Indeed, the basic idea of the sampling theorem is reported [8] to go back as far as to Borel (1897), or even to Cauchy (1841). The theorem was introduced to information theory by Shannon in 1949, see [6]. In the meanwhile, numerous 
generalizations of the theorem have been worked out. For example, in practical communication engineering applications one usually applies a generalization of the sampling theorem which improves the convergence of the sampling expansion at the price of a slight oversampling, typically on the order of $10 \%$. For standard references see e.g. [7, 8]. For recent work see, e.g., [9, 11, 12] and references therein. The ability to completely recover continuous functions from discrete samples, through the sampling theorem, is being applied ubiquitously in fields ranging from music on CDs and scientific data taking to pure mathematics where it is used, for example, to turn certain sums into easier-to-evaluate integrals.

Our aim here, as outlined in [3], is to suitably generalize Shannon sampling theory for application in the description of Planck scale physics in curved spacetime. To this end the goal is to find a sampling theorem that can accommodate variable densities of degrees of freedom, i.e. varying "bandwidths" and "Nyquist rates". In the literature, several methods for non-equally spaced sampling are known, such as Gaussian and Lagrangian interpolation, interlaced sampling, or Kramer's, Papoulis' or Paley-Wiener's sampling theorems, see, e.g., 8, 9. These, however, are either specialized or do not provide a direct handle on the time-varying bandwidth. Indeed, while it is clear, intuitively, that the bandwidth of a class of signals can be varying in time, it is surprisingly difficult to give a satisfactory definition of the concept of time-varying bandwidth:

For example, let us assume a signal $\phi(t)$ obeys a constant bandlimit $\omega_{\max }$. If we consider this signal on some finite interval $\left[t_{i}, t_{f}\right]$ and calculate its Fourier series on this interval then one might expect to find only frequency components smaller or equal to $\omega_{\max }$. This is, however, not the case. Indeed, for any finite bandwidth one can find signals which oscillate in a given interval arbitrarily faster than the highest Fourier component. This shows that any attempt must fail that tries to define a signal's time-varying bandwidth as the maximal frequency that it contains in a moving temporal window. There are surprising implications for quantum fields which in this sense "superoscillate", see [10].

There is a further reason for why the usual notion of bandwidth does not lend itself to generalization to obtain a notion of time-variable bandwidth: the usual definition of bandwidth as the range of the Fourier spectrum allows to derive Shannon's sampling theorem, but all Fourier series - they are needed in the proof - are necessarily equidistant.

For this reason, our approach here, as suggested in [3, is to aim instead for a generalized sampling theory based on a well-defined notion of variable Nyquist rate - the minimum rate at which samples must be taken can be variable. To this end, we will replace the use of Fourier techniques by the use of more powerful functional analytical techniques. Sets of sampling points will be spectra of self-adjoint operators: the spectra of self-adjoint operators do not have to be equidistant.

Technically, our starting point is the observation that if $\phi(t)$ is bandlimited then the signal $t \phi(t)$ obeys the same bandlimit. This means that no matter what bandwidth we choose, the corresponding class of bandlimited signals is invariant under the action 
of the time operator $T: \phi(t) \rightarrow t \phi(t)$. (This is because $T$ is the derivative operator on frequency space and if a function has support only strictly within a given interval, then so does its derivative.) The sampling points will be the spectra of the self-adjoint extensions of the time operator $T$.

Technically, the classes of signals with time-varying bandwidth are the domains of simple symmetric operators $T$ with deficiency indices $(1,1)$ whose self-adjoint extensions possess purely discrete spectra. The sampling kernel (in the special case of Eq2 this is the term of the form $\sin (t) / t$ ) is recognized as representing the matrix elements of the unitary operators which interpolate the eigenbases of the family of self-adjoint extensions of $T$. The sampling kernel can be calculated explicitly in terms of the chosen time-varying Nyquist rate or in terms of the time-varying "bandwidth", defined as the inverse of the time-varying Nyquist rate.

\section{Sampling theory in Hilbert space}

\subsection{Sampling kernels as unitary operators which interpolate self-adjoint extensions}

The basic idea in [3] is to reduce sampling problems to the study of a certain class of operators, namely the class of simple symmetric operators, $T$, with deficiency indices $(1,1)$ whose self-adjoint extensions $T(\alpha)$ have purely discrete spectra $\left\{t_{n}(\alpha)\right\}$. Here, $\alpha \in[0,2 \pi]$ is a real parameter which parametrizes the $U(1)$-family of self-adjoint extensions $T(\alpha)$. We denote their eigenvalues and eigenvectors by $t_{n}(\alpha)$ and $\mathbf{t}_{n}(\alpha)$, i.e.

$$
T(\alpha) \mathbf{t}_{n}(\alpha)=t_{n}(\alpha) \mathbf{t}_{n}(\alpha)
$$

As we will see, each such operators possess the property that the spectra $\left\{t_{n}(\alpha)\right\}$ of its self-adjoint extensions are covering the real line exactly once. As a consequence, after fixing the phases of the eigenvectors, each vector $\phi$ in the domain of $T$ is uniquely represented as a function

$$
\phi(t)=(\mathbf{t}, \phi) .
$$

This immediately provides the connection to sampling theory:

Any vector $\phi$ is fully determined by its coefficients in one Hilbert basis, and its coefficients in any arbitrary Hilbert basis can then be calculated. Here, if a Hilbert space vector's coefficients in the Hilbert basis $\left\{\mathbf{t}_{n}(\alpha)\right\}$ of one self-adjoint extension of $T$ are known, then the coefficient of that vector $\phi$ with any arbitrary eigenvector $\mathbf{t}$ of any arbitrary self-adjoint extension of $T$ can be calculated. Therefore, each function $\phi(t)=(\mathbf{t}, \phi)$ is already fully determined for all $t$ if only its values on one of the 
spectra are given. For any arbitrary fixed choice of $\alpha$, we obtain the reconstructionfrom-samples formula

$$
\phi(t)=\sum_{n} G\left(t, t_{n}(\alpha)\right) \phi\left(t_{n}(\alpha)\right),
$$

where the sampling kernel $G\left(t, t_{n}(\alpha)\right)$ reads

$$
G\left(t, t_{n}(\alpha)\right)=\left(\mathbf{t}, \mathbf{t}_{n}(\alpha)\right)
$$

We have merely inserted a resolution of the Hilbert space identity. The sampling kernel is therefore identified as consisting of the matrix elements of the unitaries which connect the eigenbases of the various self-adjoint extensions.

In order to make this observation into a practical sampling theorem it will be necessary to establish the relation between the sampling lattices, i.e. the family of spectra $\left\{t_{n}(\alpha)\right\}$, and the time-varying bandwidth $\omega_{\max }(t)$. This will be done in Sec 5 using the inverse relation between the bandwidth and the Nyquist sampling rate. Before, however, let us further clarify the underlying functional analytic structure.

\subsection{Simple symmetric operators}

We saw that the new and very general sampling theorem follows if we can show that for each simple symmetric operator $T$ with deficiency indices $(1,1)$ whose self-adjoint extensions have purely discrete spectra, the family of spectra $\left\{t_{n}(\alpha)\right\}$ of its self-adjoint extensions $T(\alpha)$ covers all of $\mathbb{R}$ exactly once.

To this end, let $T$ be a closed symmetric operator on a dense domain $D_{T}$ in a complex Hilbert space $H$ :

$$
(\phi, T \phi) \in \mathbb{R} \quad \forall \phi \in D_{T}
$$

Let us choose the scalar product to be antilinear in the first factor. Recall that $T$ is called simple symmetric, see e.g. [13, if it is not self-adjoint, and if it does not have any self-adjoint restriction to an invariant subspace. Note that all symmetric operators are closable.

As we indicated above, the case of most interest here is that of simple symmetric operators $T$ which have deficiency indices $(1,1)$, i.e. for which:

$$
\operatorname{dim}\left((T \pm i 1) D_{T}\right)=1
$$

It is well known that each such operator has a $U(1)$-family of self-adjoint extensions $T(\alpha)$, where $\alpha$ labels the self-adjoint extensions, e.g. $\exp (i \alpha) \in U(1)$. We consider here only such $T$ with self-adjoint extensions whose spectra are discrete.

Note that for finite deficiency indices all self-adjoint extensions have the same continuous spectrum. Thus, if one of the self-adjoint extension has a purely discrete spectrum, so do all self-adjoint extensions. As a consequence, the spectral kernel of each such 
operator $T$ is empty. This is because on the one hand any continuous part in its spectral kernel would also exist in the spectrum of its self-adjoint extensions. On the other hand, a simple symmetric operator also cannot have any discrete part in its spectral kernel, since this would contradict its simplicity ( $T$ would then have self-adjoint restrictions to eigenspaces). Therefore, for $T$, each real number $\lambda \in \mathbb{R}$ is a point of regular type, in the terminology of [13], i.e.:

$$
\exists k(\lambda)>0 \quad \forall \phi \in D_{T}: \quad\|(T-\lambda 1) \phi\| \geq k(\lambda)\|\phi\|
$$

It is known that, generally, if $\lambda$ is a real point of regular type for a simple symmetric operator $T$ with deficiency indices $(n, n)$, then there exists a self-adjoint extension $T(\alpha)$ for which $\lambda$ is an eigenvalue of multiplicity $n$. Thus, $\lambda$ is also an eigenvalue of $T^{*}$, of multiplicity at least $n$. On the other hand, if $T$ is any symmetric operator with finite and equal deficiency indices $(n, n)$, and if $\lambda \in \mathbb{R}$ is not in its point spectrum, then the multiplicity of the eigenvectors of $T^{*}$ to the eigenvalue $\lambda$ does not exceed the deficiency index $n$. Thus, if $\lambda$ is a real point of regular type of the symmetric operator $T$ then the degeneracy of the eigenvalues of $T^{*}$ is indeed exactly the deficiency index $n$.

Here, we are considering operators with deficiency indices $(1,1)$ for which the entire real line consists of points of regular type. Thus, for each $t \in \mathbb{R}$ there exists a selfadjoint extension, $T(\alpha)$, for which $t$ is an eigenvalue. Further, each real number is a nondegenerate eigenvalue of $T^{*}$. The overlap of the domain of two self-adjoint extensions is the domain of $T$. Thus, no eigenvector of $T^{*}$ can be in the domains of two self-adjoint extensions.

We can therefore conclude that for each simple symmetric operator $T$ with deficiency indices $(1,1)$ whose self-adjoint extensions have purely discrete spectra, the family of spectra $\left\{t_{n}(\alpha)\right\}$ of its self-adjoint extensions $T(\alpha)$ covers all of $\mathbb{R}$ exactly once. As we explained above, this fact directly yields the sampling theorem of Eqs 6 7. Note that, since we are working in a complex Hilbert space but wish to sample real-valued and continuous signals, we will have to prove below that it is possible to choose the phases of the eigenvectors $\{\mathbf{t}\}$ such that the sampling kernel is real and continuous.

\section{Time-varying bandwidths}

\subsection{Defining the time-varying bandwidth as a time-varying Nyquist rate}

Different choices of simple symmetric operators $T$ lead to sampling formulas for different classes of signals. Let us now identify the time-varying bandwidths of these classes of signals, as a function of the associated family of spectra $\left\{t_{n}(\alpha)\right\}$. As discussed above, it is impossible to define the notion of time-varying bandwidth in terms of the signals' Fourier transforms. Our strategy is, therefore, to define a time-varying bandwidth 
$\omega_{\max }(t)$ directly through a time-varying Nyquist rate, namely through a time-varying sampling lattice spacing function $s(t)$. We can then define $\omega_{\max }(t)=1 / 2 s(t)$. To this end, we need to show that for the spectra of the self-adjoint extensions of $T$ the lattice spacing $s(t)$ is indeed a well defined function of time.

To see this, note that each family of lattices $\left\{t_{n}(\alpha)\right\}$ is parametrizable such that the $t_{n}(\alpha)$ are differentiable, strictly monotonic functions of $\alpha$, obeying, say, $t_{n}(\alpha+2 \pi)=$ $t_{n+1}(\alpha)$. We will choose the $U(1)$-parametrization as $e^{i \alpha}$. The $t_{n}(\alpha)$ are then strictly monotonic since, as we saw, each eigenvalue of $T^{*}$ is nondegenerate and no eigenvector can be in the domain of two self-adjoint extensions.

Crucially, the strict monotonicity of the $t_{n}(\alpha)$ implies that, for a given family $\left\{t_{n}(\alpha)\right\}$, there is exactly one lattice interval centered around each point $t$. Let us denote the length of this interval by $s(t)$. Thus, if

$$
t=\frac{t_{n+1}(\alpha)+t_{n}(\alpha)}{2}
$$

for some $\alpha$, then we set

$$
s(t):=t_{n+1}(\alpha)-t_{n}(\alpha) .
$$

For each such operator $T$, the lattice spacing is therefore a well-defined function of time $s(t)$, i.e. we arrive at a well-defined time-varying Nyquist rate. Through $s(t)$, we may now define the time-varying bandwidth as

$$
\omega_{\max }(t):=\frac{1}{2 s(t)}
$$

Vice versa, given $\omega_{\max }(t)$, the family $\left\{t_{n}(\alpha)\right\}$ can be reconstructed, up to trivial reparametrization of $\alpha$, through

$$
t_{n}(\alpha)=t-\frac{1}{4 \omega_{\max }(t)}
$$

and

$$
t_{n+1}(\alpha)=t+\frac{1}{4 \omega_{\max }(t)} .
$$

It is, therefore, equivalent to specify the family of spectra $\left\{t_{n}(\alpha)\right\}$ or to specify the time-varying Nyquist rate $s(t)$, or to specify the bandwidth curve $\omega_{\max }(t)$.

The conventional characterization of the bandwidth as the width of the Fourier spectrum becomes applicable only when the bandwidth is locally approximately constant. We note that whenever the usual Fourier-based characterization of the bandwidth is applicable then it yields $\omega_{\max }:=1 / 2 s$ and it therefore agrees with our definition. 


\subsection{Bandlimited functions form the domain of a simple sym- metric time operator $T$}

Technically, we are characterizing a class of bandlimited signals as an analytic domain of the "Time operator" $T$ : $T \phi(t)=t \phi(t)$. In this way we were able to generalize the notion of bandwidth to time-varying bandwidths. There is a simple argument for why a class of bandlimited signals should be an analytic domain of $T$ (i.e. a domain on which $T$ can be applied an arbitrarily finitely many times). To this end, we need to begin with precise definitions in the case of constant bandwidth of what we mean by bandlimited functions and what we mean by strictly bandlimited functions:

We define the class $B_{\omega_{\max }}$ of strictly bandlimited signals with bandwidth $\omega_{\max }$ as consisting of all those signals whose Fourier transform $\tilde{\phi}(\omega)$ has the properties that all its derivatives $\partial_{\omega}^{n} \tilde{\phi}(\omega)$ exist and are square integrable for all $n$, and that there exists a $\omega_{0}(\phi)<\omega_{\max }$ so that

$$
\tilde{\phi}(\omega)=0 \quad \text { for all }|\omega|>\omega_{0}(\phi)
$$

We define the scalar product of two signals through

$$
\left(\phi_{1}, \phi_{2}\right)=\int_{-\omega_{\max }}^{\omega_{\max }} d \omega \tilde{\phi}_{1}(\omega)^{*} \tilde{\phi}_{2}(\omega)
$$

The closure of $B_{\omega_{\max }}$ with respect to the norm induced by Eq17 yields the Hilbert

space $H_{\omega_{\max }}:=\overline{B_{\omega_{\max }}}$ which we call the space of all bandlimited signals. (What we really mean is of course a space of physical fields that obey an ultraviolet cutoff).

Now we observe that if a signal $\phi(t)$ is strictly bandlimited, i.e. obeys Eq[16, then also the signal $T \phi(t)=t \phi(t)$ is strictly bandlimited and obeys Eq16. This is because the Fourier transform of the multiplication operator $T: T \phi(t)=t \phi(t)$ is the differentiation operator. Clearly the action of the differentiation operator action changes the support of the spectrum at most infinitesimally. Thus, if the signals Fourier transform has support strictly only inside the bandwidth interval, then also the derivative of the signals Fourier transform has support strictly only within the bandwidth interval.

Crucially, the fact that multiplying a signal $\phi(t)$ with the time variable, $\phi(t) \rightarrow t \phi(t)$, affects the bandwidth of the signal only infinitesimally, is independent of which bandwidth has been chosen. This means that, independently of the chosen bandwidth, the strictly bandlimited signals form an analytic domain of the time operator.

For this reason it is clear that any proper definition of time-varying bandwidths should have the property that each class of signals with time-varying bandwidth forms an analytic domain of the time operator.

Finally, we remark that, technically, we obtain $D_{T}$ from $B_{\omega_{\max }}$ by closing the operator (self-adjoint extensions are most conveniently constructed when starting from an operator which is closed), and we obtain $H_{\omega_{\max }}$ by closing the domain $D_{T}$ (or $B_{\omega_{\max }}$ ). 


\subsection{The bandwidth limits its own variability}

Intuitively, consistency conditions on the possible bandwidth curves $\omega_{\max }(t)$ are to be expected. This is because the bandwidth $\omega_{\text {max }}(t)$ poses a limit on how much signals can vary around time $t$ and the bandwidth should therefore also pose a limit on how much it, itself, the bandwidth $\omega_{\max }(t)$, can vary around time $t$. Indeed, there already follows a simple consistency condition from its definition in Eq.13.

Starting from

$$
\frac{d}{d t} \omega_{\max }(t)=-\frac{1}{2 s^{2}(t)} \frac{d s(t)}{d t}
$$

and using

$$
\frac{d s(t)}{d t}=\frac{d s(\alpha)}{d \alpha} \frac{d \alpha(t)}{d t}
$$

we calculate separately

$$
\frac{d s(\alpha)}{d \alpha}=\frac{d}{d \alpha}\left(t_{n+1}(\alpha)-t_{n}(\alpha)\right)
$$

and

$$
\frac{d \alpha(t)}{d t}=\left(\frac{d}{d \alpha} \frac{t_{n}(\alpha)+t_{n+1}(\alpha)}{2}\right)^{-1}
$$

Thus, introducing the notation

$$
t_{r}^{\prime}(\alpha):=\frac{d}{d \alpha} t_{r}(\alpha)
$$

we obtain:

$$
\frac{d}{d t} \omega_{\max }(t)=-4 \omega_{\max }^{2}(t) \frac{t_{n+1}^{\prime}(\alpha)-t_{n}^{\prime}(\alpha)}{t_{n+1}^{\prime}(\alpha)+t_{n}^{\prime}(\alpha)}
$$

Eq23 implies, in particular, a limit which the bandwidth $\omega_{\max }(t)$ imposes on how much $\omega_{\max }(t)$ itself can vary:

$$
\left|d \omega_{\max }(t) / d t\right|<4 \omega_{\max }^{2}(t)
$$

\subsection{The (varying) bandwidth limits how much a bandlimited signal can be peaked}

We will now show that if a signal $\phi(t)$ is strictly bandlimited by a constant $\omega_{\text {max }}$, and if $\phi(t)$ is sampled at the Nyquist rate, then the standard deviation $\Delta T$ of the sampled values is at least $\Delta T \geq 1 / 4 \omega_{\max }$. Thus, the smaller the bandwidth, the larger is the signals' minimum standard deviation, i.e. the less they can be peaked. A time-varying bandwidth means that the lower bound to the standard deviation of the signals' samples depends on the time $t$ around which the signal is centered (its first moment). 
We saw that for each Nyquist rate there is a whole family of sampling lattices. We will find that the standard deviation (as well as all other moments) of the sampled values of a strictly bandlimited signal do not depend on the lattice on which the samples are taken.

\section{The minimum standard deviation $\sigma_{\min }(t)$}

We have defined time-varying bandwidths as time-varying Nyquist rates. Let us now investigate in which sense such a time-varying bandwidth is limiting how much signals can vary over time. We will find that the time-varying Nyquist rate is limiting how much the signals can be peaked at different times.

Consider for example a normalized signal $\phi(t)$

$$
(\phi, \phi)=1
$$

which is centered around the time $t$ in the sense that $t$ is the expectation value of the time operator $T$, i.e. its first moment:

$$
(\phi, T \phi)=t
$$

A measure of how much the signal $\phi(t)$ is peaked around its expectation value is for example its second moment, i.e. its formal standard deviation:

$$
\Delta T(\phi)=\sqrt{\left(\phi, T^{2} \phi\right)-(\phi, T \phi)^{2}}
$$

In order to explicitly evaluate Eq[27 we can insert any one of the resolutions of the identity $1=\sum_{n} \mathbf{t}_{n}^{*} \otimes \mathbf{t}_{n}$ induced by the self-adjoint extensions $T(\alpha)$ of $T$, to obtain:

$$
(\Delta T(\phi))^{2}=\sum_{n} \phi\left(t_{n}(\alpha)\right)^{2} t_{n}^{2}(\alpha)-\left(\sum_{m} \phi\left(t_{m}(\alpha)\right)^{2} t_{m}(\alpha)\right)^{2}
$$

Thus, $\Delta T(\phi)$ is the standard deviation of the discrete set of samples of the signal $\phi(t)$ on a sampling lattice $\left\{t_{n}(\alpha)\right\}$. The standard deviation of a signal's samples $\phi\left(t_{n}(\alpha)\right)$ on the lattice $\left\{t_{n}(\alpha)\right\}$ is clearly the same as the standard deviation of the signal's samples $\phi\left(t_{n}\left(\alpha^{\prime}\right)\right)$ on any other lattice $\left\{t_{n}\left(\alpha^{\prime}\right)\right\}$ of the family.

We note, that therefore in the Hilbert space of bandlimited signals, the standard deviation $\Delta T(\phi)$ is not the usual standard deviation of a continuous function, which would be calculated in terms of integrals, but instead it is the standard deviation of the discrete set of samples of the signal, taken on a sampling lattice.

Our claim is that for the class of signals which is characterized by some time-varying Nyquist rate $s(t)$ there is a function $\sigma_{\min }(t)$ which sets a time-varying finite lower bound to the standard deviation $\Delta T$ of the samples of signals which are centered around $t$ :

$$
\Delta T(\phi) \geq \sigma_{\min }(t) \quad \text { for all } \phi \in D_{T} \text { which obey }(\phi, T \phi)=t
$$


Concretely, this means that if a normalized signal is sampled on a sampling lattice $\left\{t_{n}(\alpha)\right\}$ (where $\alpha$ is arbitrary) and if the average, or first moment, of the samples is $t=\sum_{n} \phi\left(t_{n}(\alpha)\right)^{2} t_{n}(\alpha)$ then we claim that the standard deviation of the samples is larger or equal than a finite value $\sigma_{\min }(t)$ :

$$
\sqrt{\sum_{n} \phi\left(t_{n}(\alpha)\right)^{2} t_{n}^{2}(\alpha)-\left(\sum_{m} \phi\left(t_{m}(\alpha)\right)^{2} t_{m}(\alpha)\right)^{2}} \geq \sigma_{\min }(t)
$$

Let us calculate the minimum standard deviation $\sigma_{\min }(t)$ from the Nyquist rate $s(t)$, i.e. from the bandwidth $\omega_{\max }(t)=1 / 2 s(t)$.

\section{Calculating $\sigma_{\min }(t)$}

In order to calculate $\sigma_{\min }(t)$ for strictly bandlimited signals, it will be convenient to calculate first the minimum standard deviation curve $\sigma_{\min }^{(\alpha)}(t)$ for a larger set signals, namely all those in the domain $D_{T(\alpha)}$ of a self-adjoint extension $T(\alpha)$ of $T$. (We recall that the dense set of strictly bandlimited signals is an analytic domain of $T$, i.e. they are contained in $D_{T}$ ).

In the domain $D_{T(\alpha)}$ of a self-adjoint extension $T(\alpha)$, there are eigenvectors $\mathbf{t}_{n}(\alpha)$ of $T(\alpha)$ and clearly each is centered around time $t_{n}(\alpha)$ and has vanishing standard deviation. We recall that the standard deviation vanishes exactly only for eigenvectors. Thus, in the domain $D_{T(\alpha)}$ of a given self-adjoint extension, the minimum standard deviation vanishes at the eigenvalues

$$
\sigma_{\min }^{(\alpha)}\left(t_{n}(\alpha)\right)=0
$$

On the other hand, for all vectors in $D_{T(\alpha)}$ which are centered around a point in time $\tau$ which is in between two neighboring eigenvalues, say $t_{n}(\alpha)<\tau<t_{n+1}(\alpha)$ the standard deviation cannot vanish, i.e. we expect that:

$$
\left.\sigma_{\min }^{(\alpha)}(\tau)\right) \neq 0
$$

In order to calculate the minimum standard deviation, $\sigma_{\min }^{(\alpha)}(\tau)$, we use that the most peaked signal $\phi_{\tau} \in D_{T(\alpha)}$ which is centered around $\tau$ must be a linear combination of the closest two eigenvectors, namely

$$
\phi_{\tau}=a_{1} \mathbf{t}_{n}(\alpha)+a_{2} \mathbf{t}_{n+1}(\alpha)
$$

The requirements $\left(\phi_{\tau}, T \phi_{\tau}\right)=\tau$ and $\left(\phi_{\tau}, \phi_{\tau}\right)=1$ determine the coefficients: $a_{1}=$ $\left(t_{n+1}-\tau\right) /\left(t_{n+1}-t_{n}\right)$, and $a_{2}=\left(\tau-t_{n}\right) /\left(t_{n+1}-t_{n}\right)$. The standard deviation $\Delta T\left(\phi_{\tau}\right)$ of the signal $\phi_{\tau}$ which is maximally peaked around the time $\tau$ is then easily calculated 
and yields the minimum standard deviation $\sigma_{\min }^{(\alpha)}(\tau)$ :

$$
\begin{aligned}
\sigma_{\min }^{(\alpha)}(\tau) & =\Delta T\left(\phi_{\tau}\right) \\
& =\sqrt{\left(\phi_{\tau}, T^{2} \phi_{\tau}\right)-\tau^{2}} \\
& =\sqrt{\left(\tau-t_{n}\right)\left(t_{n+1}-\tau\right)}
\end{aligned}
$$

It is clear that for each choice of $\alpha$ the minimum standard deviation $\sigma_{m i n}^{(\alpha)}$ is zero at all times $\left\{t_{n}(\alpha)\right\}$, since these are the eigenvalues of $T(\alpha)$. Eq[36 now shows that in the times between any two neighboring eigenvalues $t_{n}(\alpha)$ and $t_{n+1}(\alpha)$ the minimum standard deviation curve $\sigma_{\text {min }}^{(\alpha)}(t)$ is simply a half circle with diameter equal to the lattice spacing $s=t_{n+1}(\alpha)-t_{n}(\alpha)$.

We can now calculate the minimum standard deviation curve $\sigma_{\min }(t)$ for the for the vectors in $D_{T}$, from the knowledge of the minimum standard deviation curves $\sigma_{\min }^{(\alpha)}(t)$ of the self-adjoint extensions $T(\alpha)$.

This is because $D_{T} \subset D_{T(\alpha)}$ for all $\alpha$, and therefore the minimum standard deviation curve $\sigma_{\min }(t)$ for the vectors in the domain $D_{T}$ is bounded from below by all the minimum standard deviation curves $\sigma_{\min }^{(\alpha)}(t)$ of the self-adjoint extensions. We obtain:

$$
\sigma_{\min }(t)=\max _{\alpha \in U(1)} \sigma_{\min }^{(\alpha)}(t)
$$

Since strictly bandlimited signals are elements of $D_{T}$, this means that strictly bandlimited signals centered around time $t$ are peaked at most as much as specified by the minimum standard deviation $\sigma_{m i n}(t)$. Here, $\sigma_{m i n}(t)$ is the standard deviation of the discrete samples of the signal on (any arbitrary) one of the family of sampling lattices $\left\{t_{n}(\alpha)\right\}$ with the Nyquist rate $s(t)$.

In physical terms, this means that a finite ultraviolet cutoff of this kind, constant or varying, corresponds one-to-one to the existence of a constant or varying finite minimum to the formal uncertainty in position (or time), as mentioned above in the context of studies in quantum gravity and string theory, see [1].

\section{$\sigma_{\min }$ in the case of constant bandwidth}

In the case of a constant bandwidth, Eq 36 is readily evaluated:

For a constant bandwidth $\omega_{\max }$, the lattice spacing is $s=1 / 2 \omega_{\max }$. Thus, each curve $\sigma_{\text {min }}^{(\alpha)}(t)$ consists of half circles with radius $1 / 4 \omega_{\max }$ which stretch from eigenvalue to eigenvalue. Therefore, from Eq[36, the minimum standard deviation curve $\sigma_{\min }(t)$ for strictly bandlimited signals is a constant:

$$
\sigma_{\min }(t)=\frac{1}{4 \omega_{\max }}
$$


Let us confirm this result by explicitly solving the variational problem for finding the minimum standard deviation:

Our aim is to minimize $(\Delta T)^{2}$ for $\phi \in D_{T}$ by minimizing $\left(\phi, T^{2} \phi\right)$ while enforcing the constraints $(\phi, \phi)=1$ and $(\phi, T \phi)=t$.

To this end, we represent $T$ in Fourier space as $T=-i d / d \omega$ on strictly bandlimited signals, i.e. on signals which in Fourier space obey, in particular, the boundary condition:

$$
\tilde{\phi}\left(-\omega_{\max }\right)=0=\tilde{\phi}\left(\omega_{\max }\right)
$$

Introducing Lagrange multipliers $k_{1}, k_{2}$, the functional to be minimized is, therefore

$$
S=\int_{-\omega_{\max }}^{\omega_{\max }} d \omega\left\{\left(\partial_{\omega} \tilde{\phi}^{*}\right)\left(\partial_{\omega} \tilde{\phi}\right)+k_{1}\left(\tilde{\phi}^{*} \tilde{\phi}-c_{1}\right)+k_{2}\left(-i \tilde{\phi}^{*} \partial_{\omega} \tilde{\phi}-c_{2}\right)\right\}
$$

which yields the Euler-Lagrange equation:

$$
-\partial_{\omega}^{2} \tilde{\phi}+k_{1} \tilde{\phi}-i k_{2} \partial_{\omega} \tilde{\phi}=0
$$

For each value of a $T$-expectation value $t$, there exists exactly one (up to phase) normalized solution which also obeys the boundary condition, namely:

$$
\tilde{\phi}_{t}(\omega)=\frac{1}{\sqrt{2 \pi \omega_{\max }}} \cos \left(\frac{\pi \omega}{2 \omega_{\max }}\right) e^{2 \pi i t \omega}
$$

The standard deviations $\Delta T\left(\phi_{t}\right)$ of these solutions are straightforward to calculate in Fourier space, to obtain

$$
\Delta T\left(\phi_{t}\right)=\frac{1}{4 \omega_{\max }} \quad \text { for all } t
$$

which indeed coincides with our previous result, Eq 37 for the minimum standard deviation $\sigma_{\min }$ in the class of strictly bandlimited signals of constant bandwidth $\omega_{\max }$.

\section{Relation to superoscillations}

For the special case when the bandwidth is constant, the property of strictly bandlimited signals to obey a minimum standard deviation may appear to be not more and not less intuitive than the conventional belief that bandlimited signals are slow-varying, namely that bandlimited signals are varying at most as fast as their highest Fourier component.

Let us emphasize, however, that the conventional belief that bandlimited signals cannot vary faster than their fastest Fourier component does not hold true. This has been pointed out in particular in [14, 15], where counterexamples have been given 
and named "superoscillations". In 15, Berry quotes I. Daubechi in explaining that phenomena related to superoscillations have also been known to occur as instabilities in oversampling. For related work, see also e.g. [16].

On the other hand, our finding that strictly bandlimited signals obey a minimum standard deviation is true for all strictly bandlimited signals, without exceptions. Superoscillations are naturally accommodated in this framework in the sense that strictly bandlimited signals can contain arbitrarily sharp spikes, and that they can therefore be locally varying arbitrarily faster than their highest Fourier component, while still obeying the standard deviation constraint, see [17].

\section{$6 \quad$ Types of sampling lattices}

\subsection{Unbounded and semibounded sampling lattices}

In those cases where the lattices of sampling times are ranging from $-\infty$ to $+\infty$, the operator $T$ and its self-adjoint extensions are fully unbounded operators.

Since simple symmetric operators can be semibounded, it is also possible to choose sampling lattices which are merely semibounded, i.e. which range from $-\infty$ to some finite largest sampling time $t_{N}$, or of course from some finite sampling time to $+\infty$. However, we found above, on functional analytical grounds, that the family of spectra always covers all of $\mathbb{R}$, i.e. the sampling formula always allows to reconstruct $\phi(t)$ for all $t \in \mathbb{R}$. It appears to be paradoxical that signals can be sampled on a semibounded lattice but recovered for all $t \in \mathbb{R}$. Nevertheless, it is a fact that simple symmetric operators can be semi-bounded and that in such cases $\phi(t)$ can still be reconstructed for all $t \in \mathbb{R}$ from its values sampled on a semibounded lattice.

The resolution to the paradox is that the bandwidth curve $\omega_{\max }(t)$ rapidly decays after the last sampling point $t_{N}$. This means that the class of signals which are sampled in such cases is a class of signals which quickly become constant in the interval from $t_{N}$ to infinity.

To see this, assume that the spectrum of the self-adjoint extension $T(0)$ is, say, bounded from above by possessing a largest eigenvalue $t_{N}$. We recall that each real number appears as an eigenvalue in one of the self-adjoint extensions of $T$. This means that as $\alpha$ runs through the family of self-adjoint extensions, the highest eigenvalue $t_{N}(\alpha)$ of their respective spectra moves from $t_{N}(0)=t_{N}$ to infinity, $t_{N}(\alpha) \rightarrow+\infty$ for $\alpha \rightarrow 2 \pi$, while the next to highest eigenvalue behaves as $t_{N-1}(\alpha) \rightarrow t_{N}$.

Thus, while $\alpha$ runs through the family of self-adjoint extensions, the center $t=$ $\left(t_{N}(\alpha)-t_{N-1}(\alpha)\right) / 2$ of the interval between the last two eigenvalues moves to infinity, and thereby the spacing $s(t)=t_{N}(\alpha)-t_{N-1}(\alpha)$ of this interval diverges. Thus, the bandwidth $\omega_{\max }(t)=1 / 2 s(t)$ indeed decays like $\omega_{\max }(t) \rightarrow 1 / t$ between the last point of the sampling lattice and infinity.

We will show in Sec 6.3 that this phenomena can be used to sample signals on 
lattices which are not actually finite lattices, but which are effectively finite lattices for all practical purposes.

\subsection{No finite sampling lattices}

Before we discuss sampling on effectively finite sampling lattices, let us convince ourselves that there are no actually finite lattices of sampling times:

To this end, we recall that we are defining the class of signals to be sampled as the representation of the multiplication operator $T: \quad T \phi(t)=t \phi(t)$ on the space of functions for which the scalar product

$$
\left(\phi_{1}, \phi_{2}\right):=\sum_{n} \phi_{1}^{*}\left(t_{n}(\alpha)\right) \phi_{2}\left(t_{n}(\alpha)\right)
$$

is independent of $\alpha$, where $\left\{t_{n}(\alpha)\right\}$ are the spectra of a family of self-adjoint extensions.

For example, the Shannon sampling formula is now the expansion of signals

$$
\phi(t)=\sum_{n} c_{n}^{(\alpha)} b_{n}^{(\alpha)}(t)
$$

with coefficients

$$
c_{n}^{(\alpha)}=\phi\left(t_{n}(\alpha)\right)
$$

in the Hilbert basis of functions:

$$
b_{n}^{(\alpha)}(t)=\frac{\sin \left[2 \pi\left(t-t_{n}(\alpha)\right) \omega_{\max }\right]}{2 \pi\left(t-t_{n}(\alpha)\right) \omega_{\max }}
$$

The basis functions $b_{n}^{(\alpha)}(t)$ are orthogonal with respect to the scalar product given by Eq43. Here, $\left\{t_{n}(\alpha)\right\}$ is the family of lattices with the equidistant lattice points defined as in Eq 3 through $t_{n}(\alpha)=\alpha+n / 2 \omega_{\max }$. We recall that for vectors in the Hilbert space, the scalar product Eq43 yields the same result for any arbitrary choice of $\alpha$.

Here, and also in the case of generic nonequidistant sampling lattices $\left\{t_{n}(\alpha)\right\}$, the $\alpha$-independence of the scalar product implies the existence of isometries between the $l^{2}$-Hilbert spaces over each of the lattices of the family $\left\{t_{n}(\alpha)\right\}$. The matrix elements of these isometries then form the sampling kernel $G$.

This means that the signals are solutions to the equation

$$
\phi\left(t_{m}\left(\alpha^{\prime}\right)\right)=\sum_{n} G\left(t_{m}\left(\alpha^{\prime}\right), t_{n}(\alpha)\right) \phi\left(t_{n}(\alpha)\right)
$$

for all $\alpha$ and $\alpha^{\prime}$.

In addition, the signals $\phi$ form a representation of $T$, i.e. they also obey

$$
t_{m}\left(\alpha^{\prime}\right) \phi\left(t_{m}\left(\alpha^{\prime}\right)\right)=\sum_{n} G\left(t_{m}\left(\alpha^{\prime}\right), t_{n}(\alpha)\right) t_{n}(\alpha) \phi\left(t_{n}(\alpha)\right)
$$


for all $\alpha$ and $\alpha^{\prime}$. Assume now that the family $\left\{t_{n}(\alpha)\right\}$ consists of finite lattices, say with $N$ lattice sites. The sampling kernel $G$ is then a $N \times N$-dimensional matrix with matrix elements $G_{m n}\left(\alpha^{\prime}, \alpha\right):=G\left(t_{m}\left(\alpha^{\prime}\right), t_{n}(\alpha)\right)$. In this case, Eqs 4748 would yield the following equation for the sampling kernel matrix $G=G_{n m}\left(\alpha^{\prime}, \alpha\right)$ :

$$
\left(\begin{array}{ccc}
t_{1}\left(\alpha^{\prime}\right) & & 0 \\
& \ddots & \\
0 & & t_{N}\left(\alpha^{\prime}\right)
\end{array}\right)=G\left(\begin{array}{ccc}
t_{1}(\alpha) & & 0 \\
& \ddots & \\
0 & & t_{N}(\alpha)
\end{array}\right) G^{-1}
$$

It is clear that this equation cannot be solved in finite dimensions, since the adjoint action of any isometric matrix $G$ would be spectrum preserving. This simply reflects the functional analytic fact that finite dimensional symmetric operators are automatically self-adjoint, i.e. that only unbounded operators can be simple symmetric.

\subsection{Effectively finite sampling lattices}

In practical applications in engineering it is only possible to use finite lattices to sample actual signals, i.e. the theoretically infinite lattices are cut off.

The generalized sampling theorem allows us to perform the cutoff of lattices in a simple and controlled way. Namely, outside the finite time span, say $\left[t_{l o}, t_{u p}\right]$ in which actual sampling takes place, we are free to choose arbitrarily large lattice spacings. By choosing very large lattice spacings outside the interval $\left[t_{l o}, t_{u p}\right]$ we achieve that the bandwidth quickly decays to zero outside the interval. More precisely, the bandwidth decays like $1 /\left|t-t_{u p}\right|$ and $1 /\left|t-t_{l o}\right|$ to the left and right of the boundaries of the interval $\left[t_{l o}, t_{u p}\right]$, meaning that we are sampling signals which quickly become constant outside the interval. Since the signals are in the Hilbert space and are therefore square summable, they indeed decay to the constant zero. This shows that while the unboundedness of the operator $T$ is a mathematical necessity this does not affect in any essential way the sampling properties of the signals (or fields) that are based on finite sets of samples. After we develop the details of the sampling theorem we will come back to effectively finite sampling lattices in Sec 8

\section{Calculating the sampling kernel}

\subsection{Embedding a self-adjoint operator into a $U(1)$ - family of self-adjoint operators}

We begin by specifying a lattice of sampling times $\left\{t_{n}\right\}$ on which signals are to be sampled and we define the self-adjoint operator $T(0)$ which has this lattice as its spectrum. 
Our strategy is to define a self-adjoint operator $T(0)$ with the lattice $\left\{t_{n}\right\}$ as its spectrum, then to use the data $\left\{t_{n}^{\prime}\right\}$ to specify a simple symmetric restriction $T$ of $T(0)$, and finally to construct its $U(1)$ - family of self-adjoint extensions $T(\alpha)$. The scalar product of their eigenvectors yields the desired sampling kernel $G\left(t, t_{n}\right)$.

To this end, consider the self-adjoint operator

$$
T(0):=\sum_{n} t_{n} \mathbf{t}_{n} \otimes \mathbf{t}_{n}^{*}
$$

on its domain $D_{T(0)}$. Here, $\mathbf{t}_{n}$ denotes the eigenvector to the eigenvalue $t_{n}$, and $\mathbf{t}_{\mathbf{n}}^{*}$ denotes the dual vector. The Cayley transform of $T(0)$ is a unitary operator

$$
U(0)=\sum_{n} \frac{t_{n}-i}{t_{n}+i} \mathbf{t}_{n} \otimes \mathbf{t}_{n}^{*}
$$

which is defined on the entire Hilbert space $H=\bar{D}_{T(0)}$. We restrict $U(0)$ to a simple isometric operator

$$
S=\left.U(0)\right|_{H \ominus C \mathbf{v}_{+}}
$$

by taking from its domain one dimension spanned by a normalized vector $\mathbf{v}_{+} \in H$. The inverse Cayley transform then yields a simple symmetric operator $T$ on $D_{T}$

$$
D_{T}=\left\{\phi \in D_{T(0)} \mid\left(\mathbf{v}_{+},(T(0)+i) \phi\right)=0\right\}
$$

iff the so-defined $D_{T}$ is dense and contains no eigenvectors. In the $\left\{\mathbf{t}_{n}\right\}$-basis, the condition $\left(\mathbf{v}_{+},(T(0)+i) \phi\right)=0$ reads $\sum_{n}\left(\mathbf{v}_{+}, \mathbf{t}_{n}\right)\left(t_{n}+i\right)\left(\mathbf{t}_{n}, \phi\right)=0$. Thus, for $D_{T}$ to be dense, $\mathbf{v}_{+}$must be chosen such that

$$
\sum_{n}\left|\left(\mathbf{v}_{+}, \mathbf{t}_{n}\right)\left(t_{n}+i\right)\right|^{2}=\text { divergent }
$$

since otherwise all of $D_{T}$ would be orthogonal to $\mathbf{w}:=\sum_{\mathrm{n}}\left(\mathrm{t}_{\mathrm{n}}+\mathrm{i}\right)\left(\mathbf{v}_{+}, \mathbf{t}_{\mathrm{n}}\right) \mathbf{t}_{\mathrm{n}}$, which would then be a vector in $H$.

Further, the condition that there are no eigenvectors in $D_{T}$ implies that $\mathbf{v}_{+}$must be chosen such that $\left(\mathbf{v}_{+}, t_{n}\right) \neq 0$ for all $n$. The second deficiency space is of course spanned by $\mathbf{v}_{-}=U(0) \mathbf{v}_{+}$. Now a $U(1)$-family of self-adjoint extensions $T(\alpha)$ of $T$ is obtained as the inverse Cayley transforms of the unitary extensions $U(\alpha)$ of $S$ :

$$
U(\alpha)=\left(1-\mathbf{v}_{-} \otimes \mathbf{v}_{-}^{*}+e^{i \alpha} \mathbf{v}_{-} \otimes \mathbf{v}_{-}^{*}\right) U(0)
$$

The choice of a spectrum $\left\{t_{n}\right\}=\left\{t_{n}(0)\right\}$ and a deficiency vector $\mathbf{v}_{+}$thus determines a family of self-adjoints $T(\alpha)$ and unitaries $U(\alpha)$ with their eigenbases $\left\{\mathbf{t}_{n}(\alpha)\right\}$ (up to phases). The functions $t_{n}(\alpha)$ are strictly monotonic since each eigenvalue of $T^{*}$ is nondegenerate and no eigenvector can be in the domain of two self-adjoint extensions. 


\subsection{Calculating the $t$-dependence of the sampling kernel}

The aim is to calculate the sampling kernel, i.e. the scalar products of the eigenvectors of the unitary and self-adjoint extensions. To this end, let $\mathbf{t}_{r}(\alpha)$ be a normalized eigenvector of the operators $U(\alpha)$ and $T(\alpha)$. Then:

$$
\begin{aligned}
\frac{t_{r}(\alpha)-i}{t_{r}(\alpha)+i} \quad\left(\mathbf{t}_{n}, \mathbf{t}_{r}(\alpha)\right) & =\left(\mathbf{t}_{n}, U(\alpha) \mathbf{t}_{r}(\alpha)\right) \\
& =\left(\mathbf{t}_{n},\left(1+\left(e^{i \alpha}-1\right) \mathbf{v}_{-} \otimes \mathbf{v}_{-}^{*}\right) U(0) \mathbf{t}_{r}(\alpha)\right) \\
& =\frac{t_{n}-i}{t_{n}+i}\left(\mathbf{t}_{n}, \mathbf{t}_{r}(\alpha)\right)+\left(e^{i \alpha}-1\right)\left(\mathbf{t}_{n}, \mathbf{v}_{-}\right)\left(\mathbf{v}_{+}, \mathbf{t}_{r}(\alpha)\right)
\end{aligned}
$$

Thus,

$$
\left(\mathbf{t}_{n}, \mathbf{t}_{r}(\alpha)\right)=\frac{f_{n} N\left(t_{r}(\alpha)\right)}{t_{r}(\alpha)-t_{n}}
$$

where we defined

$$
N\left(t_{r}(\alpha)\right):=\frac{t_{r}(\alpha)+i}{2 i}\left(e^{i \alpha}-1\right)\left(\mathbf{v}_{+}, \mathbf{t}_{r}(\alpha)\right)
$$

and

$$
f_{n}:=\left(t_{n}+i\right)\left(\mathbf{t}_{n}, \mathbf{v}_{-}\right)
$$

In this way we have separated the $t_{r}(\alpha)$-dependence of the sampling kernel in the form of $N\left(t_{r}(\alpha)\right)$ from its $n$-dependence through the coefficients $f_{n}$. It is now straightforward to calculate $N\left(t_{r}(\alpha)\right)$, i.e. the $t_{r}(\alpha)$-dependence of the sampling kernel by using the normalization condition:

$$
1=\sum_{n}\left(\mathbf{t}_{r}(\alpha), \mathbf{t}_{n}\right) \quad\left(\mathbf{t}_{n}, \mathbf{t}_{r}(\alpha)\right)=\sum_{n} \frac{f_{n}^{*} f_{n} N^{*}\left(t_{r}(\alpha)\right) N\left(t_{r}(\alpha)\right)}{\left(t_{r}(\alpha)-t_{n}\right)^{2}}
$$

Thus, for all $t$ in the family $\left\{t_{r}(\alpha)\right\}$, i.e. for all $t \in \mathbb{R}$, there holds

$$
|N(t)|=\left(\sum_{n} \frac{f_{n}^{*} f_{n}}{\left(t-t_{n}\right)^{2}}\right)^{-1 / 2}
$$

\subsection{Calculating the $n$-dependence of the sampling kernel}

In order to calculate the coefficients $f_{n}$, we begin by noting that

$$
\begin{aligned}
\left(\mathbf{t}_{n}, \mathbf{v}_{-}\right) & =\left(\mathbf{t}_{n}, U(0) \mathbf{v}_{+}\right) \\
& =\sum_{m}\left(\mathbf{t}_{n}, U(0) \mathbf{t}_{m}\right)\left(\mathbf{t}_{m}, \mathbf{v}_{+}\right)
\end{aligned}
$$




$$
\begin{aligned}
& =\sum_{m}\left(\mathbf{t}_{n}, \mathbf{t}_{m}\right) \frac{t_{m}-i}{t_{m}+i}\left(\mathbf{t}_{m}, \mathbf{v}_{+}\right) \\
& =\frac{t_{n}-i}{t_{n}+i}\left(\mathbf{t}_{n}, \mathbf{v}_{+}\right)
\end{aligned}
$$

so that, in addition to Eq 58 , the $f_{n}$ also obey

$$
f_{n}=\left(t_{n}-i\right)\left(\mathbf{t}_{n}, \mathbf{v}_{+}\right)
$$

We will now show that $t_{n}^{\prime} \propto f_{n}^{*} f_{n}$, i.e., more precisely, that $d t_{n}(\alpha) n /\left.d \alpha\right|_{\alpha=0}=f_{n}^{*} f_{n} / 2$.

To this end, inserting a resolution of the identity in terms of the $\left\{\mathbf{t}_{n}\right\}$-eigenbasis into Eq 57 yields

$$
N\left(t_{r}(\alpha)\right)=\frac{t_{r}(\alpha)+i}{2 i}\left(e^{i \alpha}-1\right) \sum_{n}\left(\mathbf{v}_{+}, \mathbf{t}_{n}\right) \quad\left(\mathbf{t}_{n}, \mathbf{t}_{r}(\alpha)\right)
$$

Inserting Eq[56] and Eq[61, we obtain

$$
N\left(t_{r}(\alpha)\right)=\frac{t_{r}(\alpha)+i}{2 i}\left(e^{i \alpha}-1\right) \sum_{n} \frac{f_{n}^{*} f_{n} N\left(t_{r}(\alpha)\right)}{\left(t_{n}+i\right)\left(t_{r}(\alpha)-t_{n}\right)}
$$

Thus:

$$
\frac{2 i}{e^{i \alpha}-1}=\sum_{n} \frac{f_{n}^{*} f_{n}\left(t_{r}(\alpha)+i\right)}{\left(t_{n}+i\right)\left(t_{r}(\alpha)-t_{n}\right)}
$$

Let us calculate separately the imaginary and the real part of Eq64. The imaginary part yields:

$$
\frac{1}{e^{i \alpha}-1}+\frac{1}{e^{-i \alpha}-1}=\frac{1}{2 i} \sum_{n} f_{n}^{*} f_{n} \frac{(t(\alpha)+i)\left(t_{n}-i\right)-(t(\alpha)-i)\left(t_{n}+i\right)}{\left(t_{n}^{2}+1\right)\left(t(\alpha)-t_{n}\right)}
$$

and thus,

$$
-1=\sum_{n} \frac{-f_{n}^{*} f_{n}}{t_{n}^{2}+1}
$$

Using Eq 61, we see that Eq [66 simply expresses the normalization condition for $\mathbf{v}_{+}$. On the other hand, the real part of Eq 64 yields:

$$
\begin{gathered}
\frac{1}{e^{i \alpha}-1}-\frac{1}{e^{-i \alpha}-1}=\frac{1}{2 i} \sum_{n} f_{n}^{*} f_{n}\left(\frac{t(\alpha)+i}{\left(t_{n}+i\right)\left(t(\alpha)-t_{n}\right)}+\frac{t(\alpha-i}{\left(t_{n}-i\right)\left(t(\alpha)-t_{n}\right)}\right) \\
i \frac{e^{-i \alpha}-e^{i \alpha}}{2-e^{i \alpha}-e^{-i \alpha}}=\frac{1}{2} \sum_{n} f_{n}^{*} f_{n} \frac{(t(\alpha)+i)\left(t_{n}-i\right)+(t(\alpha)-i)\left(t_{n}+i\right)}{\left(t_{n}^{2}+1\right)\left(t(\alpha)-t_{n}\right)}
\end{gathered}
$$


and thus, using

$$
\begin{aligned}
\cot (\alpha / 2) & =i \frac{e^{i \alpha / 2}+e^{-i \alpha / 2}}{e^{i \alpha / 2}-e^{-i \alpha / 2}} \\
& =i \frac{e^{-i \alpha}-e^{i \alpha}}{2-e^{i \alpha}-e^{-i \alpha}}
\end{aligned}
$$

we obtain:

$$
\cot (\alpha / 2)=\sum_{n} \frac{f_{n}^{*} f_{n}\left(t_{n} t_{r}(\alpha)+1\right)}{\left(t_{n}^{2}+1\right)\left(t_{r}(\alpha)-t_{n}\right)}
$$

Since $t_{r}(\alpha) \rightarrow t_{r}$ for $\alpha \rightarrow 0$, Eq 69 finally yields:

$$
\left.\frac{d t_{r}(\alpha)}{d \alpha}\right|_{\alpha=0}=\frac{f_{r}^{*} f_{r}}{2}
$$

\subsection{The deficiency vector $\mathrm{v}_{+}$is parametrized by the coeffi- cients $f_{n}$}

Through Eq61, the deficiency vector $\mathbf{v}_{+}$is parametrized by the coefficients $f_{n}$. Thus, the constraints on the choice of $\mathbf{v}_{+}$are in one-to-one correspondence to constraints on the choice of coefficients $f_{n}$, namely

$$
f_{n} \neq 0 \text { forall } n, \quad \sum_{n} \frac{f_{n}^{*} f_{n}}{1+t_{n}^{2}}=1 \text { and } \sum_{n} f_{n}^{*} f_{n}=\text { divergent } .
$$

Clearly, such $f_{n}$ only exist if $\left|t_{n}\right| \rightarrow \infty$ for $n \rightarrow \infty$ and/or for $n \rightarrow-\infty$. This reflects the functional analytical fact that only semibounded or fully unbounded symmetric operators can have nonzero deficiency indices.

We can now use Eq 70 to express the conditions on $\mathbf{v}_{+}$, as conditions on the coefficients $\left\{t_{n}^{\prime}\right\}$. We obtain as the constraints on the data $\left\{t_{n}\right\}$ and $\left\{t_{n}^{\prime}\right\}$ :

$$
\begin{aligned}
& \quad t_{n+1}>t_{n}, \text { and } t_{n}^{\prime}>0 \text { for all } n, \\
& \left|t_{n}\right| \rightarrow \infty \text { for } n \rightarrow+\infty \text { and/or for } n \rightarrow-\infty
\end{aligned}
$$

and

$$
\sum_{n} \frac{t_{n}^{\prime}}{1+t_{n}^{2}}=\text { finite, } \quad \sum_{n} t_{n}^{\prime}=\text { divergent. }
$$

\subsection{Realness and differentiability of the sampling kernel}

While we are working in a complex Hilbert space, the aim in engineering would be to sample real-valued signals. In the case of physical fields, we may want to work with

charged, i.e., complex-valued scalar fields, but we will certainly also want to be able to work with real-valued scalar fields. 
We therefore have to deal with the fact that the representation of vectors $\phi$ of the Hilbert space as functions $\phi(t)=(\mathbf{t}, \phi)$ a priori depends on the arbitrary choice of the phases of the vectors $\mathbf{t}$. Correspondingly, also the sampling kernel $\left(\mathbf{t}, \mathbf{t}_{n}\right)$ depends on the choice of these phases. For the sampling of real-valued signals we therefore need to show that it is possible to choose the phases of the vectors $\mathbf{t}$ such that if a signal is real-valued on one sampling lattice $\left\{t_{n}\right\}$, then it is real-valued for all $t$. This means that we need to show that it is possible to choose the phases such that the sampling kernel is real-valued.

In order to derive the appropriate choice of phases, we reconsider the sampling kernel Eq 56 .

$$
\left(\mathbf{t}_{n}, \mathbf{t}\right)=\frac{f_{n} N(t)}{t-t_{n}}
$$

which holds for all $t \neq t_{n}$, while, of course,

$$
\left(\mathbf{t}_{n}, \mathbf{t}_{n}\right)=1
$$

We observe that the denominator in Eq75] goes linearly to zero whenever $t$ approaches one of the $t_{n}$ of the lattice. Nevertheless, the absolute value of the sampling kernel does not diverge since, as follows from Eq 60 simultaneously also the numerator linearly approaches zero as $t$ approaches $t_{n}$.

In particular, whenever $t$ passes through a $t_{n}$ the denominator in Eq 75 changes sign. Thus, in order for the sampling kernel to be continuous, it is necessary that also $N(t)$ changes sign at the points $t_{n}$, i.e. as it passes through a zero.

Indeed, the definition of $N(t)$ in Eq.57 shows that it is possible to choose the phases of the vectors $\mathbf{t} \neq \mathbf{t}_{n}$ such that

$$
N\left(t_{r}(\alpha)\right)=(-1)^{r}\left|N\left(t_{r}(\alpha)\right)\right|
$$

This choice of phases ensures that the sampling kernel is real and continuous for all $t \neq t_{n}$, and that, for all $n$, both limits of the sampling kernel $\left(\mathbf{t}_{n}, \mathbf{t}\right)$ for $t \rightarrow t_{n}$, from above and below, coincide. However, in order to obtain that the sampling kernel is continuous at the times $t_{n}$, we still need to ensure that those limits also coincide with the value $\left(\mathbf{t}_{n}, \mathbf{t}_{n}\right)=1$ set by the normalization condition Eq.76. Therefore, since $N(t)$ changes sign at each $t_{n}$, also the $f_{n}$ must have alternating signs:

$$
f_{n}=(-1)^{n}\left|f_{n}\right|
$$

We still have the freedom to choose the phases of the vectors $\mathbf{t}_{n}$. Indeed, using Eq 61 , we can choose the phases of these vectors so that Eq78 holds.

We remark that the choice of phases to obtain the sampling kernel is unique up to a global phase: The sampling kernel is a scalar product of the vectors $\mathbf{t}$ and therefore the same real and differentiable sampling kernel is obtained if all vectors $\mathbf{t}$ are multiplied by some constant overall phase $c$. Indeed, the definitions of $N(t)$ and $f_{n}$ show that 
this amounts to multiplying $N(t)$ with $c$ and multiplying $f_{n}$ with $c^{*}$, so that the phase cancels out in the expression for the sampling kernel.

We have therefore shown that and how the phases of the vectors $\mathbf{t}$ can be chosen so that the sampling kernel is real and continuous, to obtain:

$$
\left(\mathbf{t}_{n}, \mathbf{t}_{r}(\alpha)\right)=(-1)^{n+r} \frac{\left|f_{n}\right|\left|N\left(t_{r}(\alpha)\right)\right|}{t_{r}(\alpha)-t_{n}}
$$

The sign factor can be written, equivalently, as

$$
(-1)^{z\left(t_{r}(\alpha), t_{n}\right)}
$$

where $z\left(t_{r}(\alpha), t_{n}\right)$ is the number of sampling points $t_{i}$ in the interval $\left[t_{r}(\alpha), t_{n}\right]$ if $t_{r}(\alpha)<$ $t_{n}$ or the number of $t_{i}$ in the interval $\left[t_{n}, t\right]$ if $t_{n}<t_{r}(\alpha)$, and $z=0$ if $t_{n}=t_{r}(\alpha)$.

Since each $t \in \mathbb{R}$ occurs in the family of lattices $\left\{t_{r}(\alpha)\right\}$ we can now express the sampling kernel in the general form:

$$
\left(\mathbf{t}_{n}, t\right)=(-1)^{z\left(t, t_{n}\right)} \frac{\left|f_{n}\right||N(t)|}{t-t_{n}}
$$

Finally, using or results Eqs 70 57, which express $\left|f_{n}\right|$ and $|N(t)|$ in terms of the data $\left\{t_{n}\right\}$ and $\left\{t_{n}^{\prime}\right\}$ we obtain:

$$
\left(\mathbf{t}_{n}, \mathbf{t}\right)=(-1)^{z\left(t, t_{n}\right)} \frac{\sqrt{t_{n}^{\prime}}}{t-t_{n}}\left(\sum_{m} \frac{t_{m}^{\prime}}{\left(t-t_{m}\right)^{2}}\right)^{-1 / 2}
$$

\subsection{Calculating the full sampling kernel $G\left(t, t^{\prime}\right)$, which yields all sampling lattices and $\omega_{\max }(t)$ for all $t$}

So-far we have only calculated the sampling kernel $\left(\mathbf{t}, \mathbf{t}_{n}\right)$ for one sampling lattice $\left\{t_{n}\right\}=\left\{t_{n}(0)\right\}$ of the family of sampling lattices. It is straightforward to calculate the general sampling kernel:

$$
\begin{aligned}
\left(\mathbf{t}_{r}(\alpha), \mathbf{t}_{s}\left(\alpha^{\prime}\right)\right) & =\sum_{n}\left(\mathbf{t}_{r}(\alpha), \mathbf{t}_{n}\right)\left(\mathbf{t}_{n}, \mathbf{t}_{s}\left(\alpha^{\prime}\right)\right) \\
& =\sum_{n} \frac{(-1)^{r+s} t_{n}^{\prime}}{\left(t_{r}(\alpha)-t_{n}\right)\left(t_{s}\left(\alpha^{\prime}\right)-t_{n}\right) \sqrt{\left(\sum_{m} \frac{t_{m}^{\prime}}{\left(t_{r}(\alpha)-t_{m}\right)^{2}}\right)\left(\sum_{m} \frac{t_{m}^{\prime}}{\left(t_{s}\left(\alpha^{\prime}\right)-t_{m}\right)^{2}}\right)}}
\end{aligned}
$$

or, in simplified notation, for arbitrary times $t, \tau \in \mathbb{R}$ :

$$
(\mathbf{t}, \tau)=\sum_{\mathrm{m}} \frac{(-1)^{\mathrm{z}(\mathrm{t}, \tau)} \mathrm{t}_{\mathrm{n}}^{\prime}}{\left(\mathrm{t}-\mathrm{t}_{\mathrm{n}}\right)\left(\tau-\mathrm{t}_{\mathrm{n}}\right) \sqrt{\left(\sum_{\mathrm{m}} \frac{\mathrm{t}_{\mathrm{m}}^{\prime}}{\left(\mathrm{t}-\mathrm{t}_{\mathrm{m}}\right)^{2}}\right)\left(\sum_{\mathrm{m}} \frac{\mathrm{t}_{\mathrm{m}}^{\prime}}{\left(\tau-\mathrm{t}_{\mathrm{m}}\right)^{2}}\right)}}
$$


The significance of this equation is that, given one sampling lattice $\left\{t_{n}\right\}=\left\{t_{n}(0)\right\}$ and its derivative $\left\{t_{n}^{\prime}\right\}=\left\{\frac{d}{d \alpha} t_{n}(0)\right\}$, Eq 83 allows us reconstruct the entire family $\left\{t_{n}(\alpha)\right\}$ of sampling lattices, up to reparametrization of $\alpha$. This is because each of the sampling lattices consists of the eigenvalues of orthogonal vectors, i.e. of vectors whose scalar product vanishes:

For any $t_{0} \in \mathbb{R}$ there exists a self-adjoint extension $T(\alpha)$ and some index $n$ so that $t_{0}=t_{n}(\alpha)$. The entire sampling lattice $\left\{t_{n}(\alpha)\right\}$ then consists of $t_{0}$ and all $t$ for which $\left(\mathbf{t}_{0}, \mathbf{t}\right)=0$ :

$$
\left\{t_{n}(\alpha)\right\}=\left\{t_{0}\right\} \cup\left\{t \mid\left(\mathbf{t}_{0}, \mathbf{t}\right)=0\right\}
$$

To this end, for generic data $\left\{t_{n}\right\}$ and $\left\{t_{n}^{\prime}\right\}$ the zeros of the general sampling kernel in $\mathrm{Eq} 83$ can at least be found numerically. Thus, all lattices of the family $\left\{t_{n}(\alpha)\right\}$ can be calculated, in principle, from the data $\left\{t_{n}\right\},\left\{t_{n}^{\prime}\right\}$ which enter the sampling theorem.

In turn, once all sampling lattices are known, also the curve $\omega_{\max }(t)$ can be constructed explicitly for all $t$, as explained above.

\subsection{Other sampling lattices}

In the new sampling theorem, the class of signals to be sampled is chosen by specifying one sampling lattice $\left\{t_{n}\right\}$ and its derivative, $\left\{t_{n}^{\prime}\right\}$ and by thereby specifying the timevarying bandwidth curve $\omega_{\max }(t)$. The sampling theorem allows us to recover the signals of this class from their values at any one of the sampling lattices from the family of sampling lattices which is determined by the data $\left\{t_{n}\right\}$ and $\left\{t_{n}^{\prime}\right\}$.

In fact, the same class of signals, with bandwidth specified by the data $\left\{t_{n}\right\}$ and $\left\{t_{n}^{\prime}\right\}$, can also be sampled on a large variety of other sampling lattices, by using a suitably modified sampling formula.

To this end, assume that $\left\{\tilde{t}_{n}\right\}$ is any sampling lattice for which there exists a linear transformation $K$, such that:

$$
\sum_{r} K_{s r}\left(\tilde{\mathbf{t}}_{r}, \mathbf{t}_{m}\right)=\delta_{s m}
$$

Applied to

$$
\sum_{m}\left(\tilde{\mathbf{t}}_{r}, \mathbf{t}_{m}\right)\left(\mathbf{t}_{m}, \phi\right)=\left(\tilde{\mathbf{t}}_{r}, \phi\right)
$$

this yields

$$
\begin{aligned}
\left(\mathbf{t}_{n}, \phi\right) & =\sum_{m, r} K_{n, r}\left(\tilde{\mathbf{t}}_{r}, \mathbf{t}_{m}\right)\left(\mathbf{t}_{m}, \phi\right) \\
& =\sum_{r} K_{n, r}\left(\tilde{\mathbf{t}}_{r}, \phi\right)
\end{aligned}
$$

Inserting this result into $(\mathbf{t}, \phi)=\sum_{n}\left(\mathbf{t}, \mathbf{t}_{n}\right)\left(\mathbf{t}_{n}, \phi\right)$, we obtain the sampling formula for sampling on the lattice $\left\{\tilde{\mathbf{t}}_{n}\right\}$ :

$$
(\mathbf{t}, \phi)=\sum_{n, r}\left(\mathbf{t}, \mathbf{t}_{n}\right) K_{n, r}\left(\tilde{\mathbf{t}}_{r}, \phi\right)
$$




\section{Summary of the new sampling method}

The new sampling method allows us to continuously increase and lower the sampling rate over time in order to sample signals with a correspondingly time-varying bandwidth. For engineering purposes, this allows one to economize on data collection whenever the bandwidth is temporarily low and to increase the sampling rate whenever the bandwidth is temporarily high. This is the basis of the data compression method patented in 4 .

The mathematical background is based on the observation that the action of the "time" operator $T: T \phi(t)=t \phi(t)$ can only infinitesimally change the spectrum of bandlimited signals (because it acts as differentiation in Fourier space), independently of the chosen bandwidth. We were able, therefore, to identify classes of signals with time-varying bandwidth as analytic domains of simple symmetric multiplication operators $T: \phi(t) \rightarrow t \phi(t)$. (In the case of fields in spacetime, we may think, equivalently, of $T$ as a position coordinate.)

In particular, we found that, as in the case of constant bandwidth, also in the case of a time-varying bandwidth, there exists a family of lattices $\left\{t_{n}(\alpha)\right\}$ of sampling times at which the signals can be measured and recovered for all $t$. We found that the lattice spacings of these sampling lattices, together, form a well-defined function of time $s(t)$. This yielded a precise definition of time-varying bandwidths as time-varying Nyquist rates, i.e. as a time-dependence of the spacings $s(t)$ of sampling lattices. We defined $\omega_{\max }(t)=1 / 2 s(t)$.

We also discussed that, contrary to intuition, a finite bandwidth does not imply that signals vary "at most as fast as their highest Fourier component". We found that instead a true property of finite bandwidth signals is that they obey a lower bound to how much they can be peaked, in the sense that the standard deviation of the samples $\phi\left(t_{n}(\alpha)\right)$ (for arbitrary fixed $\alpha$ ) of strictly bandlimited signals is bounded from below.

More generally, we found that classes of signals with time-varying bandwidths obey a time-varying lower bound on their standard deviation, i.e. they consist of signals which, if centered around time $t$, have standard deviations bounded from below by a positive time-dependent function $\sigma_{\max }(t)$.

\subsection{How to use the sampling theorem}

Concretely, the sampling theorem requires as input data first the choice of one sampling lattice $\left\{t_{n}\right\}$. The choice of one sampling lattice largely determines the Nyquist rate and therefore the time-varying bandwidth $\omega_{\max }(t)$. This is because by giving one sampling lattice the Nyquist rate $s(t)$, i.e. the lattice spacing, is defined already for a discrete set of intervals. This means that the bandwidth is already defined at a discrete set of times, namely at the times $\tau_{n}=\left(t_{n}+t_{n+1}\right) / 2$, i.e. at the center of each lattice interval, 
through the inverse of the spacing:

$$
\omega_{\max }\left(\tau_{n}\right)=\frac{1}{2 s(t)}=\frac{1}{2\left(t_{n+1}-t_{n}\right)}
$$

We also found that the bandwidth cannot vary much between these points:

$$
\left|\frac{d}{d t} \omega_{\max }(t)\right|<4 \omega_{\max }(t)^{2} .
$$

The sampling theorem requires a further set of input data, $\left\{t_{n}^{\prime}\right\}$, to fine-tune the bandwidth curve by specifying the derivative of the bandwidth at those points, namely through:

$$
\frac{d}{d t} \omega_{\max }\left(\tau_{n}\right)=-4 \omega_{\max }\left(\tau_{n}\right)^{2} \frac{\left(t_{n+1}^{\prime}-t_{n}^{\prime}\right)}{\left(t_{n+1}^{\prime}+t_{n}^{\prime}\right)}
$$

We showed that the bandwidth curve $\omega_{\max }(t)$ thereby becomes determined for all $t$. We recall that by embedding the sampling lattice $\left\{t_{n}\right\}$ as the lattice $\left\{t_{n}(0)\right\}$ into the family of sampling lattices $\left\{t_{n}(\alpha)\right\}$ which is determined by (and determines) the bandwidth $\omega_{\max }(t)$, the auxiliary data can be identified as the derivative of the lattice: $\left\{t_{n}^{\prime}\right\}=\left\{d /\left.d t t_{n}(\alpha)\right|_{\alpha=0}\right\}$.

After choosing sampling times $\left\{t_{n}\right\}$, which already largely determine the bandwidth, and auxiliary data $\left\{t_{n}^{\prime}\right\}$, which fine-tune and fix the bandwidth curve $\omega_{\max }(t)$ for all $t$, the sampling theorem then allows to recover all signals of the class with this bandwidth through the sampling formula:

$$
\phi(t)=\sum_{n} G\left(t, t_{n}\right) \phi\left(t_{n}\right)
$$

with the sampling kernel $G\left(t, t_{n}\right)$ :

$$
G\left(t, t_{n}\right)=(-1)^{z\left(t, t_{n}\right)} \frac{\sqrt{t_{n}^{\prime}}}{t-t_{n}}\left(\sum_{m} \frac{t_{m}^{\prime}}{\left(t-t_{m}\right)^{2}}\right)^{-1 / 2}
$$

The number of eigenvalues $t_{n}$ in a given interval defines what we call the local density of degrees of freedom and variable bandwidth. Taking samples at the so-defined Nyquist rate expands functions in terms of an orthonormal basis of functions. The reconstruction is, therefore, stable in the sense that small perturbations in the sample values lead to small perturbations in the reconstructed function. Stability of the reconstruction is important since sample values are generally subject to noise and/or to quantum uncertainties. For the case of conventional Shannon sampling it is known that reconstruction from samples taken at a lower rate than the Nyquist rate is possible, in principle, but must be unstable, as Landau proved in [18.

Here too, we may choose to use the data $\left\{t_{n}\right\},\left\{t_{n}^{\prime}\right\}$ merely in order to fix the bandwidth curve $\omega_{\max }(t)$ and then decide to sample the so-specified class of signals on 
a different sampling lattice. Indeed, we found that the same class of signals can be sampled also on any other lattice, say $\left\{\tilde{t}_{n}\right\}$, for which $K_{r, s}=\left(G\left(\tilde{t}_{r}, t_{s}\right)\right)_{r, s}$ is invertible. The sampling formula then reads:

$$
\phi(t)=\sum_{n, m} G\left(t, t_{n}\right) K_{n, m}^{-1} \phi\left(\tilde{t}_{m}\right)
$$

The spacing of these sampling lattices $\left\{\tilde{t}_{n}\right\}$ may be larger than the spacing of the eigenvalues of the self-adjoint extensions of $T$. Since we are covering conventional Shannon sampling as a special case, see Sec 8.2, it is to be expected that reconstruction is necessarily unstable if the samples are taken at a lower rate than the density of the degrees of freedom. It should be interesting to prove this in generality.

Finally, let us briefly address the case of engineering applications, where all sampling lattices are finite.

We recall from Sec 6.2 that finite sampling lattices do not occur in the theoretical framework. Technically, the reason is that the underlying time operator $T$ can only be simple symmetric if it is unbounded. Thus, in principle, in Eqs 9192 the indices $n$ and $m$ which label the sampling times $t_{n}$ must run through a countably infinite index set, e.g. the natural numbers or the integers. Therefore, a truncation error appears to be unavoidable.

Our generalized sampling theorem allows us, however, to freely choose the sampling points $\left\{t_{n}\right\}$, as long as the sequence $\left\{t_{n}\right\}$ is divergent. This freedom can be used to sample on effectively finite sampling lattices, say $\left\{t_{n}\right\}_{n=1}^{n=N}$ :

To this end, we extend the finite sampling lattice $\left\{t_{n}\right\}_{n=1}^{n=N}$ on which we wish to sample to an infinite sampling lattice by adding an infinite number of further sampling points. These additional sampling points we are free to choose for example at very very large $t$, where the signals have negligible support. Thus, these sampling points do not contribute to either of the sums in Eqs 9192. In practise, we can therefore leave Eqs 9192 unchanged and we simply restrict the sums over $n$ and $m$ to the finite sampling lattice $\left\{t_{n}\right\}_{n=1}^{n=N}$.

As discussed in Sec6.3, the class of signals which is being sampled in this case has a bandwidth curve $\omega_{\max }(t)$ which decays as $1 / t$ outside the interval $\left[t_{1}, t_{N}\right]$ in which the samples are taken.

\subsection{Recovering conventional Shannon sampling as a special case}

The standard Shannon Sampling Formula is to arise as a special case. The class of signals with constant bandwidth $\omega_{\max }$ is characterized by the family of lattices $\left\{t_{n}(\alpha)\right\}$ with $t_{n}(\alpha)=\alpha+n / 2 \omega_{\max }$. Choose the sampling lattice $\left\{t_{n}\right\}=\left\{t_{n}(0)\right\}$. Its derivative 
$\left\{t_{n}^{\prime}\right\}$ is $t_{n}^{\prime}=1$. Thus, Eq 92 yields the sampling formula:

$$
\phi(t)=\left(\sum_{m=-\infty}^{+\infty} \frac{1}{\left(t-t_{m}\right)^{2}}\right)^{-1 / 2} \sum_{n=-\infty}^{+\infty} \frac{(-1)^{z\left(t, t_{n}\right)}}{t-t_{n}} \phi\left(t_{n}\right)
$$

Since this equation must coincide with the Shannon formula, Eq, 2, we can conclude that

$$
\frac{\pi^{2}}{\sin ^{2}(\pi x)}=\sum_{n=-\infty}^{+\infty} \frac{1}{(x-n)^{2}}
$$

which provides an example of a series expansion deduced with the new method. Note that setting $x=1 / 2$ we obtain the expansion $\pi=\sqrt{\sum_{n=-\infty}^{+\infty}(n-1 / 2)^{-2}}$.

\section{On the relation to coherent states and wavelets}

\subsection{One - and two parameter overcomplete, continuous and normalizable resolutions of the identity}

We are here obtaining resolutions of the identity in terms of an overcomplete and continuously parametrized set of normalizable vectors,

$$
\begin{aligned}
1 & =\frac{1}{2 \pi} \int_{0}^{2 \pi} d \alpha \sum_{n} \mathbf{t}_{n}^{*}(\alpha) \otimes \mathbf{t}_{n}(\alpha) \\
& =\frac{1}{2 \pi} \int_{-\infty}^{+\infty} d t \frac{d \alpha}{d t} \mathbf{t}^{*} \otimes \mathbf{t} .
\end{aligned}
$$

In this sense, our expansion is similar to coherent states and wavelet expansions. The main difference is that while we arrive at a one-parameter resolution of the identity in the Hilbert space, coherent states and wavelets, see e.g. [19], are typically characterized, analogously, by two-parameter resolutions of the identity. In this context, it will be interesting to follow the analogous construction to the one here, but for time-operators with higher deficiency indices.

The resolution of the identity obtained in Eq94 implies that we are in fact dealing with a reproducing kernel Hilbert space. Namely, the signals $\phi$ obey

$$
(\mathbf{t}, \phi)=\frac{1}{2 \pi} \int_{-\infty}^{+\infty} d \tau \frac{d \alpha}{d t}(\tau)(\mathbf{t}, \tau)(\tau, \phi)
$$

so that, with the reproducing kernel

$$
R(t, \tau):=(\mathbf{t}, \tau) \frac{\mathrm{d} \alpha}{\mathrm{dt}}(\tau)
$$

we obtain:

$$
\phi(t)=\frac{1}{2 \pi} \int_{-\infty}^{+\infty} d \tau R(t, \tau) \phi(\tau)
$$




\subsection{Calculation of the reproducing kernel}

In Sec 7.6 we calculated the sampling kernel $(\mathbf{t}, \tau)$ for all $t, \tau$ in terms of the input data $\left\{t_{n}\right\},\left\{t_{n}^{\prime}\right\}$. In order to determine the reproducing kernel it remains to calculate $d \alpha / d t$. To this end, we solve Eq 69 for $\alpha$, by using

$$
\cot ^{-1}(z)=\frac{i}{2} \ln \frac{i z+1}{i z-1}
$$

so that

$$
\alpha=i \ln \frac{i y(t)+1}{i y(t)-1}
$$

where

$$
y(t)=\sum_{n} t_{n}^{\prime} \frac{t t_{n}+1}{\left(t-t_{n}\right)\left(t_{n}^{2}+1\right)} .
$$

We can now calculate

$$
\frac{d \alpha}{d t}=\frac{d \alpha}{d y} \frac{d y}{d t}
$$

from

$$
\frac{d \alpha}{d y}=\frac{-2}{1+y^{2}}
$$

and

$$
\frac{d y}{d t}=\sum_{n} t_{n}^{\prime} \frac{1}{\left(t-t_{n}\right)^{2}}
$$

Thus, we obtain

$$
\frac{d \alpha}{d t}=\frac{2 \sum_{m} t_{m}^{\prime} \frac{1}{\left(t-t_{m}\right)^{2}}}{1+\left(\sum_{n} t_{n}^{\prime} \frac{t t_{n}+1}{\left(t-t_{n}\right)\left(t_{n}^{2}+1\right)}\right)^{2}}
$$

which finally yields a fully explicit expression for the reproducing kernel:

$$
R(t, \tau)=(-1)^{z(t, \tau)} \sqrt{\frac{\sum_{r} \frac{t_{r}^{\prime}}{\left(\tau-t_{r}\right)^{2}}}{\sum_{s} \frac{t_{s}^{\prime}}{\left(t-t_{s}\right)^{2}}}} \frac{\sum_{n} \frac{2 t_{n}^{\prime}}{\left(t-t_{n}\right)\left(\tau-t_{n}\right)}}{1+\left(\sum_{m} \frac{t_{m}^{\prime}\left(\tau t_{m}+1\right)}{\left.\left(\tau-t_{m}\right) t_{m}^{2}+1\right)}\right)^{2}}
$$

\section{Outlook}

In the literature, studies of quantum gravity and string theory, see [1], have suggested a natural ultraviolet cutoff in the form of a finite lower bound to the resolution of distances with a corresponding modification of the uncertainty relations. As discussed in Sec 5.4, theories that are ultraviolet cutoff in the sampling theoretic sense concretely realize such generalized uncertainty relations. A sampling theoretic ultraviolet cutoff at the Planck scale also corresponds to a finite density of degrees of freedom for physical 
fields in the very same sense that bandlimited music signals possess a finite density of degrees of freedom and a correspondingly finite information density.

This leads to the prospect that sampling theory could allow us to formulate physical theories which combine the virtues of lattice theories with the virtues of continuum theories. Fields, such as those of quantum field theoretic path integrals, could be defined over a continuous spacetime manifold or, entirely equivalently, over any lattice which is sufficiently densely spaced. A theory's ultraviolet finiteness would be displayed when formulated on one of the lattices, while the same theory's preservation of external symmetries would be displayed when written as a continuum theory.

Here, we proved sampling results for the one-dimensional case as a preparation for the generalization of Shannon sampling theory to generic curved spacetimes which has been outlined in [3] and [5]. To this end, we generalized sampling theory using the functional analysis of simple symmetric operators which have deficiency indices $(1,1)$ and whose self-adjoint extensions have purely discrete spectra. It should be straightforward to apply these functional analytic methods to simple symmetric operators with higher deficiency indices, thereby leading to sampling methods for vector- and spinor-valued fields. The very same methods should be useful for developing the details of the general sampling theory of fields on n-dimensional curved spacetimes.

It is encouraging that the mathematical language of the sampling theoretical Planck scale cutoff, namely functional analysis, is also the language of quantum theory.

Acknowledgement: The author is happy to thank John Klauder for useful criticisms.

\section{References}

[1] D.J. Gross, P.F. Mende, Nucl. Phys. B303, 407 (1988), D. Amati, M. Ciafaloni, G. Veneziano, Phys. Lett. B216 41, (1989), A. Kempf, J. Math. Phys. 35 (9), 4483 (1994), D.V. Ahluwalia, Phys. Lett. B339, 301 (1994), M.-J. Jaeckel, S. Reynaud, Phys. Lett. A185, 143 (1994), E. Witten, Phys. Today 49 (4), 24 (1996), G. Amelino-Camelia, J. Ellis, N.E. Mavromatos, D.V. Nanopoulos, Mod. Phys. Lett.A12 2029 (1997), A. Kempf , G. Mangano, Phys. Rev. D55, 7909 (1997)

[2] G.'t Hooft, Class. Quant. Grav. 16, 3263 (1999), J.D. Bekenstein, Acta Phys.Polon. B32, 3555 (2001), quant-ph/0110005, R. Bousso, Phys. Rev. Lett. 90, 121302 (2003), P.S.Custodio, J.E.Horvath, gr-qc/0305022

[3] A. Kempf, Phys. Rev. Lett. 85 : 2873 (2000)

[4] A. Kempf, Method for monitoring information density and compressing digitized signals, US patent \# 6,531,971 B2 (2003)

[5] A. Kempf, gr-qc/0310035 
[6] C. E. Shannon, Proceedings of the Institute of Radio Engineers, 37, (1) 10 (1949), C.E. Shannon, W. Weaver, The mathematical theory of communication, Univ. of Illinois Press (1949), re-issued (1998)

[7] A. J. Jerri, Proc. IEEE, 65 , (11) 1565 (1977)

[8] R. J. Marks, Introduction to Shannon Sampling and Interpolation Theory, Springer (1991)

[9] A. I. Zayed, J. Math. Anal. and Appl., 158 , 269 (1991), A. I. Zayed, J. Math. Anal. and Appl., 175 , 222 (1993)

[10] A. Kempf, P.J.S.G. Ferreira, quant-ph/0305148

[11] Y. I. Lyubarskii, K. Seip, J. Fourier Analysis and Appl., 3 , (5) 597 (1997)

[12] M. H. Annaby, Math. Meth. Appl. Sc. 21 , 361 (1998)

[13] N. I. Akhiezer, I. M. Glazman, Theory of Linear Operators in Hilbert Space, Dover Publ., N.Y. (1993)

[14] Y. Aharonov, J. Anandan, S. Popescu, L. Vaidman, Phys. Rev. Lett. 642965 (1990)

[15] M. Berry, in Proc. Intl. Conf. on Fund. Aspects of Quantum Theory, Columbia, SC, USA, 10-12 Dec. 1992 Eds. J.S. Anandan, J. L. Safko, World Scientific, Singapore (1995)

[16] M. V. Berry, J. Phys. A27 L391 (1994)

[17] A. Kempf, P.J.S.G. Ferreira, quant-ph/0305148

[18] H.J. Landau, Proc. of the IEEE, 10, 1701 (1967), H.J. Landau, Acta Mathematica, 117, 37 (1967)

[19] J. R. Klauder, Wavelet Applications VI, Proc. SPIE, 3723, 44 (1999) 